\title{
Closed-loop control of product geometry by using an artificial neural network in incremental sheet forming with active medium
}

\author{
S. Thiery ${ }^{1}$ (D) $\cdot$ M. Zein El Abdine ${ }^{1} \cdot$ J. Heger ${ }^{1} \cdot$ N. Ben Khalifa ${ }^{1,2}$
}

Received: 17 September 2020 / Accepted: 3 November 2020 / Published online: 13 November 2020

(C) The Author(s) 2020

\begin{abstract}
A strategy to adjust the product geometry autonomously through an online control of the manufacturing process in incremental sheet forming with active medium is presented. An axial force sensor and a laser distance sensor are integrated into the process setup to measure the forming force and the product height, respectively. Experiments are conducted to estimate the bulging behavior for different pre-determined tool paths. An artificial neural network is consequently trained based on the experimental data to continuously predict the pressure levels required to control the final product height. The predicted pressure is part of a closed-loop control to improve the geometrical accuracy of formed parts. Finally, experiments were conducted to verify the results, where truncated cones with different dimensions were formed with and without the closed-loop control. The results indicate that this strategy enhances the geometrical accuracy of the parts and can potentially be expanded to be implemented for different types of material and geometries.
\end{abstract}

Keywords Incremental sheet forming with active medium · Closed-loop control · Artificialneural networks · Geometricalaccuracy

\section{Introduction}

Incremental sheet forming with active medium (IFAM) is a novel manufacturing process to produce concave-convex geometrical parts in small lot sizes [1]. This process an extension of the well-known single point incremental forming (SPIF) by an active medium, which applies pressure on the bottom surface of the blank during the forming process. Depending on tool path and pressure control, concave and convex forming operations are feasible in IFAM and can be sequentially combined. Shaping complex parts requires neither intermediate turning of the blank nor a dedicated die nor a counter tool like other common incremental forming processes. Therefore, IFAM preserves the flexibility of incremental forming processes. The active medium can be gas or liquid. Ben Khalifa and Thiery [1] used pressurized air for convex forming.

S. Thiery

sebastian.thiery@leuphana.de

1 Institute of Product and Process Innovation, Leuphana University of Lüneburg, Universitätsallee 1, 21335 Lüneburg, Germany

2 Institute of Materials Research, Helmholtz-Zentrum Geesthacht, Max-Planck-Straße 1, 21502 Geesthacht, Germany
Moreover, Kumar and Kumar [2] utilized pressurized fluid for concave forming. Plastic deformation occurs only in the contact zone between tool and blank due to stress concentration. For this reason, forming forces and pressure of the active medium are comparatively low. Ben Khalifa and Thiery [1] investigated the minimum pressure that is required to enable convex forming. While machining AA1050A-H14 sheets with a thickness of $1 \mathrm{~mm}$, the relatively low minimum pressure value of 0.35 bar can lead to cracks if the forming operation is repeated multiple times at the same area. Cracks are the dominant failure mechanism of IFAM and are reported for both convex and concave forming [1, 3, 4]. On the one hand, pressure has to be adjusted during the process to avoid cracks and to limit bulging. On the other hand, a pre-defined pressure is necessary to achieve a target shape.

The manufacturing procedure in incremental sheet forming processes begins with the definition of the desired product shape by computer-aided design (CAD). Subsequently, computer-aided manufacturing (CAM) can easily generate the tool path for SPIF applications by moving the tool along the product contour [5]. This tool path can then be transferred as G-code to a CNC machine to start the forming operation. CAM solutions reach their limits if either complex parts are to be manufactured or a second tool, for example in double-sided incremental forming (DSIF), has to be considered. For this 
purpose, Ndip-Agbor et al. [6] developed specialized algorithms to ensure a quick tool path generation. Nonetheless, deviation from the target geometry can arise during the forming operation. Having no support under the sheet, SPIF has inferior accuracy than other incremental forming processes. Springback and undesired bending are the dominant phenomena that negatively affect its accuracy. Several strategies have been developed to reduce or compensate these inaccuracies. One possibility is to use a dedicated rig to define the transition between flange and part wall more clearly [7]. Another possibility is to adapt the tool path in accordance with the real deviation after a first part is manufactured [8]. Afterwards, the adapted tool path can be used to produce a second part with better accuracy. Ambrogio et al. [9] evaluate elastic springback and provide a simple approach to improve accuracy by emphasizing finite element method (FEM) as an appropriate tool to predict the part shape. The previous mentioned strategies are examples taken from a wide-ranging field of investigations that have been published on the issue of improving the geometrical accuracy in incremental forming. However, IFAM requires setpoints for the pressure of the active medium besides the tool path definitions. CAM systems are not designed in their function to calculate such setpoints and even FEM software cannot be used to determine the setpoints in reasonable time. A control system might be a feasible solution to regulate the pressure online.

The geometrical accuracy as well as other product properties are often the target of closed-loop control systems in metal forming. Uncertainties in production processes lead to deviation from a desired target value or process instability. The uncertainties might have non-repetitive behavior, for example, uneven lubrication or repetitive behavior such as tool wear [10]. Closed-loop control is one approach to cope with these uncertainties [11]. Closed-loop control systems can be distinguished into three types [12]. The equipment closed-loop control ensures that the actuators work exactly like the predefined trajectory without concerning workpiece state or product properties. The equipment is encapsulated by the online closed-loop control, which reacts to changes in the workpiece state during the manufacturing process. The offline closed loop control builds the outer shell and measures the product properties after the manufacturing process. One important element of a closed-loop control is the control model, which might be a conventional PID control or a meta-model such as artificial neural networks (ANNs) [12]. Additionally, a sensor is required to measure the workpiece state, though the integration of sensors is challenging because the accessibility of forming tools is restricted [11]. However, this does not apply for incremental forming, which does not have large dedicated dies. Allwood et al. [13] employed a camera system to detect the part shape during SPIF and implemented an online closed-loop control to adjust the tool path accordingly. Their control scheme is based on a discrete principle and measures the part shape once for each cycle. In contrast, Filice et al. [14] presented an online control based on continuous force measurement. The vertical forming force was used as an indicator for sheet thinning and process failure. If a critical state is reached, tool diameter and step down are automatically adjusted.

The recent advancements in the context of Industry 4.0 have offered an opportunity in the transformation of today's manufacturing paradigm to smart manufacturing [15]. As a result, datasets generated by modern manufacturing systems are experiencing explosive growth [15]. The former, in combination with the advancements in computational capabilities, has resulted in machine learning approaches based on ANNs gaining a lot of popularity within the manufacturing community as a whole, both in industry and academia. They are used for a wide range of applications, including tool wear monitoring and forecasting [16, 17], decision support systems [18], process parameter predictions [19], quality control [20,21], etc. They are also gaining more traction within incremental sheet forming; specifically, Khan et al. [22] used ANNs to predict local springback errors in an SPIF process and adjusted the tool path accordingly. Hartmann et al. [23] developed an automated process for which a deep neural network can generate tool paths based on the desired geometry for an incremental sheet metal free-forming process for non-complex parts. Kurra et al. [24] used the tool diameter, step depth, wall angle, feed rate and lubricant type to estimate the surface roughness using ANNs. Ambrogio et al. [25] used an ANN to predict failure in an incremental sheet forming process where the formability and the final height of the part were investigated through varying geometrical properties (wall inclination angles).

In their previous work, Ben Khalifa and Thiery [1] formed concave-convex parts while manually adjusting the pressure. As such, forming parts using constant pressure levels was not investigated. This paper aims to compare the geometrical accuracy of formed parts using constant pressure against parts formed with dynamically adjusted pressure. However, the absence of a die or a dedicated tool makes the dynamic control of the IFAM process challenging. In order to do so, a method that is capable of dealing with the intrinsically complex interrelations of the process parameters is required. Hence, mathematical modelling, in the form of ANNs is selected to control the pressure necessary to improve the geometrical accuracy and further automate the process.

The rest of the paper is structured as follows: The experimental setup is first introduced including the process definition along with its parameters. Then, the use of ANNs for offline pressure prediction is described and the full control strategy summarized. Finally, experiments are conducted to compare the results for truncated cones formed using constant and dynamically adjusted pressure. 


\section{Experimental setup and control design}

\section{Process description}

The process of manufacturing a convex truncated cone by IFAM is shown in Fig. 1. At the beginning, the pressure $p$ is applied to the bottom of the blank and the tool moves to the start position of the tool path, which is located near the center of the blank (Fig. 1a). The vertical position of the tool $z$ is defined in relation to the unpressurized blank surface and is restricted within a short distance during the process to establish contact between the tool and the blank. Subsequently, the tool follows a predefined path and moves in concentric cycles towards the margin of the blank (Fig. 1b). Two consecutive cycles have a horizontal distance $\Delta x$ between each other. The part emerges upwards with every cycle and the process is designated as positive or convex forming for this reason. The tool path is flat and the tool moves forward in horizontal direction until a desired number of cycles $n$ is completed (Fig. 1c). After removing the tool and releasing the pressure, the manufacturing process is finished.

The setup is placed on the bench of a milling machine and includes the workpiece, blank holder and tool. The workpiece is fixed by the blank holder to the pressure chamber to build a closed system. A laser distance sensor is installed by a holding device on the bottom of the pressure chamber (Fig. 2) to detect the part height. The measurable distance of this sensor reaches from a minimum of $30 \mathrm{~mm}$ to a maximum of $130 \mathrm{~mm}$. The sensor is aligned so that the laser beam coincides with the center of the blank. There is an offset distance $h_{\text {off }}$ between the sensor and the bottom surface of the blank that needs to be measured at the beginning of each manufacturing process. The sensor cable runs through a sealed cable gland to the outside of the pressure chamber. Pressurized air is used as an active medium, and its pressure can be observed by a manometer or recorded by a pressure sensor. A tube connects the air supply of the pressure chamber with a pressure control valve. The valve has a working range from 0.0 to $6.0 \mathrm{bar}$, and its setpoint can be changed during the manufacturing process. Oil is used for all experiments to reduce friction and tool wear. The tool has a hemispherical shape with a radius of $5 \mathrm{~mm}$. An axial force sensor is integrated into the tool holder, and the force can be measured even during tool rotation. The rotational speed is $60 \mathrm{rpm}$ for the present investigation and the vertical tool position is kept constant at $z=0 \mathrm{~mm}$. The tool is attached to the spindle of the milling machine, and the information about the tool position and actual NC block are sent to a measurement computer, which also collects the data of the pressure, force and laser sensor during the manufacturing process. Examples for processible materials are pure aluminum with up to $2 \mathrm{~mm}$ thickness or deep drawing steel DC04 with $1 \mathrm{~mm}$ thickness. Pure aluminum AA1050A-H24, which is in a half-hard state and has a thickness of $0.96 \mathrm{~mm}$, is used for all present experiments. The initial flow stress of this material amounts $117 \mathrm{MPa}$ in rolling direction. According to the dimensions of the pressure chamber, the blanks have a shape of $280 \mathrm{~mm} \times 280 \mathrm{~mm}$ and an area of $190 \mathrm{~mm} \times 190 \mathrm{~mm}$ inside
Fig. 1 Manufacturing process of incremental sheet forming with active medium: a first contact between tool and workpiece, $\mathbf{b}$ intermediate cycle, $\mathbf{c}$ final cycle of the manufacturing process
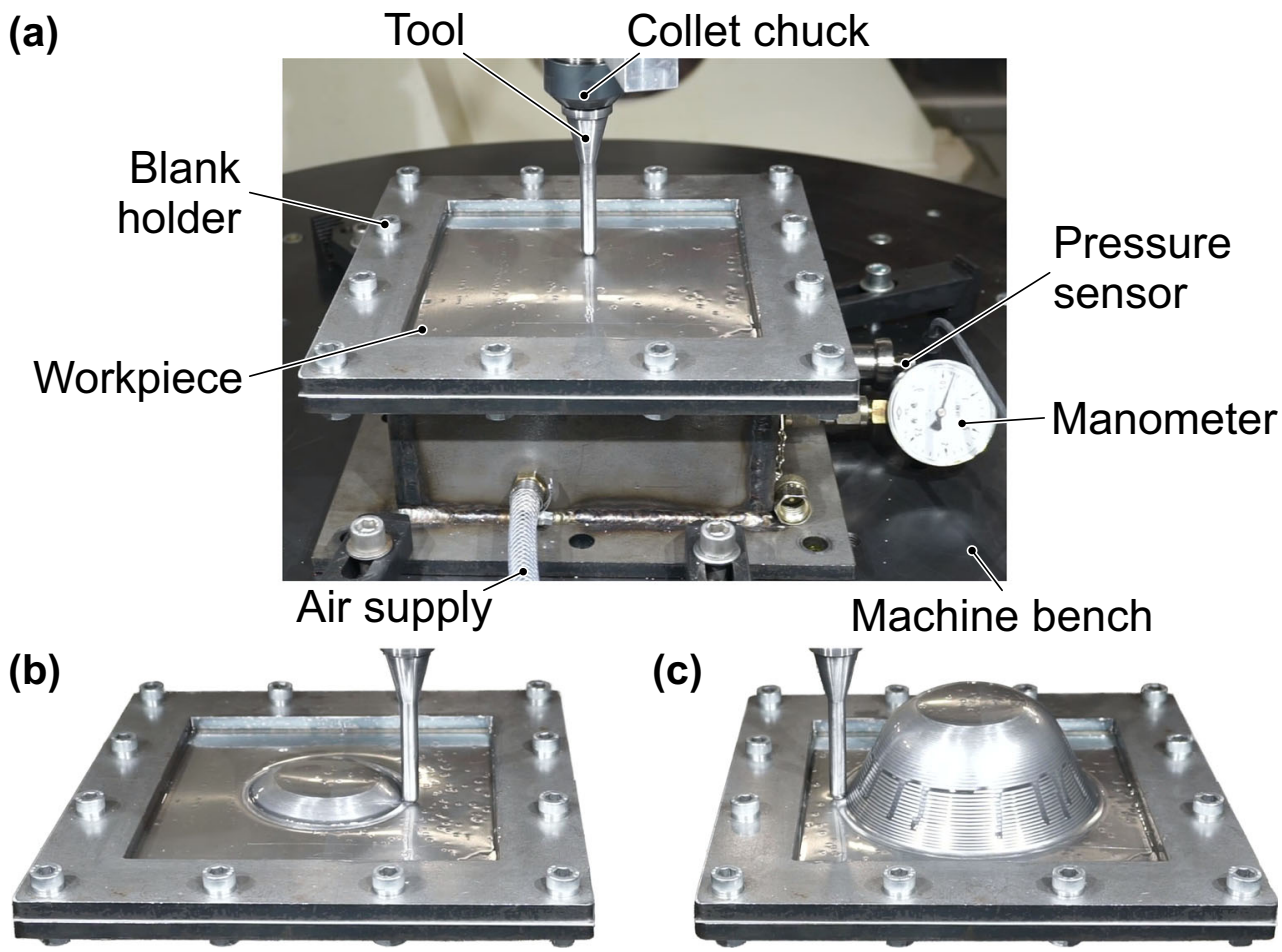

(c)

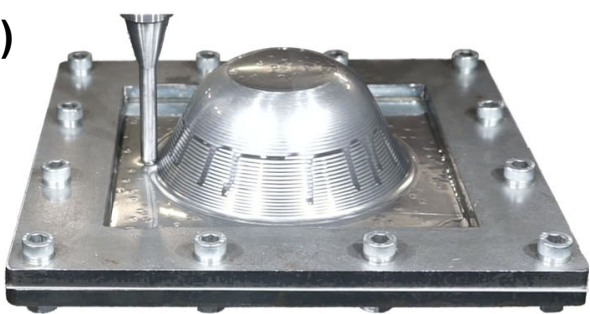


Fig. 2 Online measurement of the part height $h$ by including a laser distance sensor into the pressure chamber

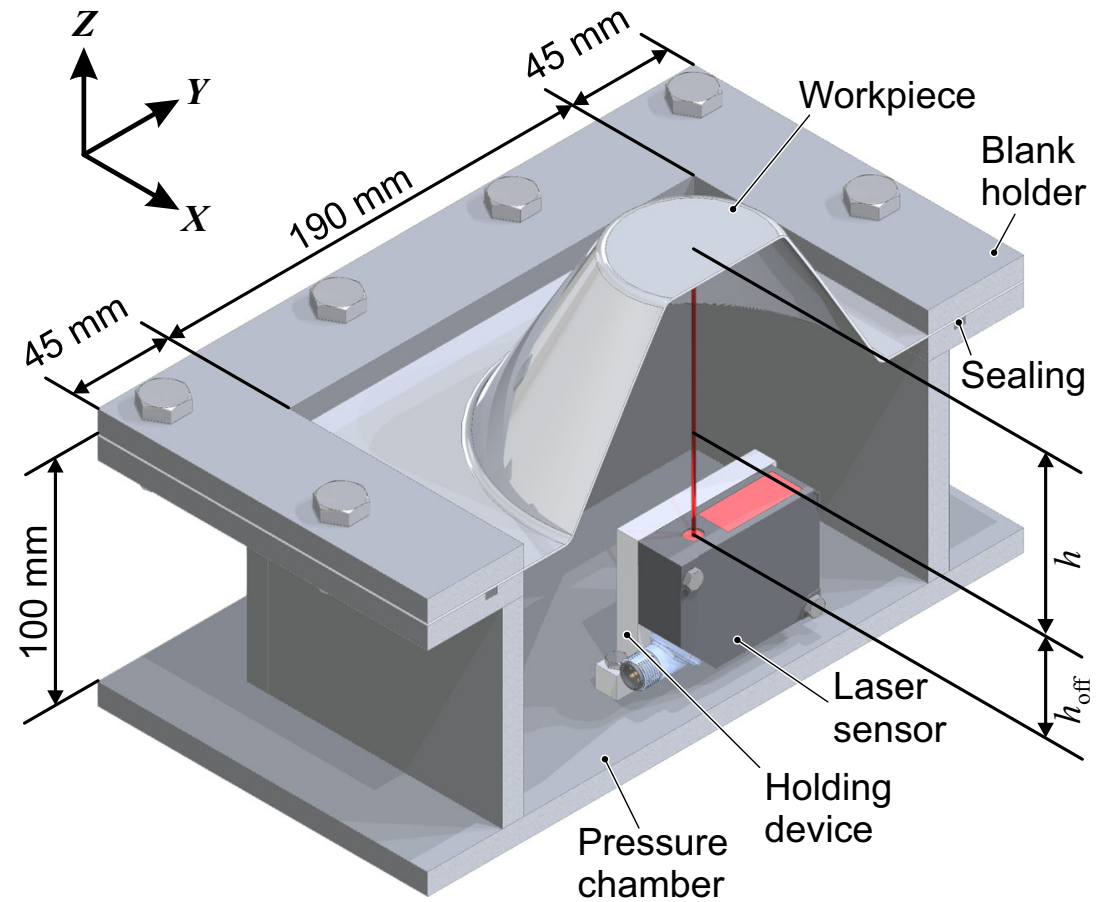

the clamping. The rolling direction of the blank is orientated parallel to the $X$-axis of the milling machine.

\section{Experimental conditions}

The part height $h$ grows during IFAM with every cycle of the tool path. Unlike SPIF, in which the tool path predefines the final geometry, the final geometry and the part height $h$ in IFAM result from the interaction of the tool path and the pressure. With the same tool path but with a different pressure level, parts with other heights or wall angles can be manufactured. Therefore, experiments are conducted to evaluate the bulging behavior enclosing height and force measurement and to generate a dataset. Five different tool paths have been defined for these experiments, of which three are shown in Fig. 3. The circular tool paths are distinguished by the number of cycles $n$ and by both the start and end position. The vertical tool positon is $z=0 \mathrm{~mm}$ and the horizontal step size is $\Delta x=1 \mathrm{~mm}$ for all paths. The feed rate is set to $f=$ $1500 \mathrm{~mm} / \mathrm{min}$ for the whole process. The pressure was held constant for each part, and was varied in increments of 0.025 bar for different parts with different paths, as can be seen in Table 1. The pressure was stepwise increased until cracks occurred. No target geometry was defined for these experiments but instead the actual height and also the vertical force were recorded with a frequency of $50 \mathrm{~Hz}$. Afterwards, the measurement protocols were analyzed for each cycle. The vertical force $F$ is then the average value from all recorded values during one cycle. The product height $h$ is defined as the average of 5 values at the end of each cycle.

\section{Artificial neural networks}

Neural networks and supervised machine learning algorithms in general learn from labeled examples and are evaluated based on how well they can generalize their learning on unseen examples. Specifically, hidden patterns and non-linear relationships within the data are learned by the algorithms. This way, the algorithms can react to yet unknown instances. In the case where the relation between the desired part shape and the process parameters is characterized by a high degree of complexity, it is integral to use algorithms which can represent and learn the interrelations among the data. The data retrieved from the experiments in Table 1 allow the training of an algorithm which can predict the pressure levels for different tool paths and geometries. ANNs consist of a collection of interconnected processing elements called neurons that are combined as layers. The first layer being the input layer, the last layer the output layer and in-between the hidden layers. The neurons process the input simultaneously through a series of matrix multiplications where the hidden layers perform nonlinear mapping between the input and output layers. The output signal of a neuron is fed to other neurons as input signals. For an input to the network $X^{m \times 1}$, the following layer's output $Y^{n \times 1}$, may be expressed by Eq. 1:

$Y=\sigma(W \cdot X+B)$

Where $W$ is an $n \times m$ weight matrix, $\sigma$ is the nonlinear activation function and $B$ a vector bias which adjusts the threshold at which the neuron gets activated [26]. Such a feed-forward neural network is trained by back-propagation with supervised 
Table 1 Testing parameters to investigate the bulging behavior

\begin{tabular}{|c|c|c|c|c|}
\hline Part & Minor radius & Major radius & Number of cycles $n$ & Pressure range \\
\hline Small & $50 \mathrm{~mm}$ & $60 \mathrm{~mm}$ & 11 & $0.475-0.625$ bar \\
\hline Middle & $45 \mathrm{~mm}$ & $65 \mathrm{~mm}$ & 21 & $0.450-0.600$ bar \\
\hline Narrow & $35 \mathrm{~mm}$ & $55 \mathrm{~mm}$ & 21 & $0.525-0.675$ bar \\
\hline Wide & $55 \mathrm{~mm}$ & $75 \mathrm{~mm}$ & 21 & $0.400-0.550$ bar \\
\hline Large & $35 \mathrm{~mm}$ & $75 \mathrm{~mm}$ & 41 & $0.425-0.575$ bar \\
\hline
\end{tabular}

learning. During training, known input-output combinations are repeatedly passed through the network to determine the loss function (error) of a given prediction. Consequently, the weights $W$ and biases $B$ are adjusted in order to minimize the loss function of the training set. If the error is too high, the weights and biases are updated. The new weights are again used for prediction, and the error is re-calculated. This process continues until the error or the number of iterations reaches a pre-determined value. The main drawback of ANNs is their tendency to overfit the learning on the training set, resulting in them not generalizing very well. Additionally, adjusting the network's hyperparameters to maximize performance can be challenging and time consuming. In order to overcome overfitting, three forms of regularization are employed:

- Dropout: Random input units are set to zero with a predefined ratio. Through dropping out subsets of features during training, overfitting is reduced.
- Validation set: While the model uses the training set to learn and fit the parameters, this fit is tested on a separate validation set.

- Early stopping: If the training error on the validation set does not decrease within the next 50 epochs, then training is halted.

Furthermore, an exhaustive search along with trial and error was performed in order to find the best set of hyperparameters suited to the dataset at hand. Specifically, the topology of the network, dropout ratio, batch size, and weight initialization have been explored and taken into consideration. The range of the parameters to be optimized with regard to topology consisted of the following:

- Hidden layers: $\{1,2,3,4\}$

- Neurons per layer: $\{10,15,20,25,50,100,125,150,200$, $250,500\}$
Fig. 3 Depiction of one quarter of the axisymmetric tool path

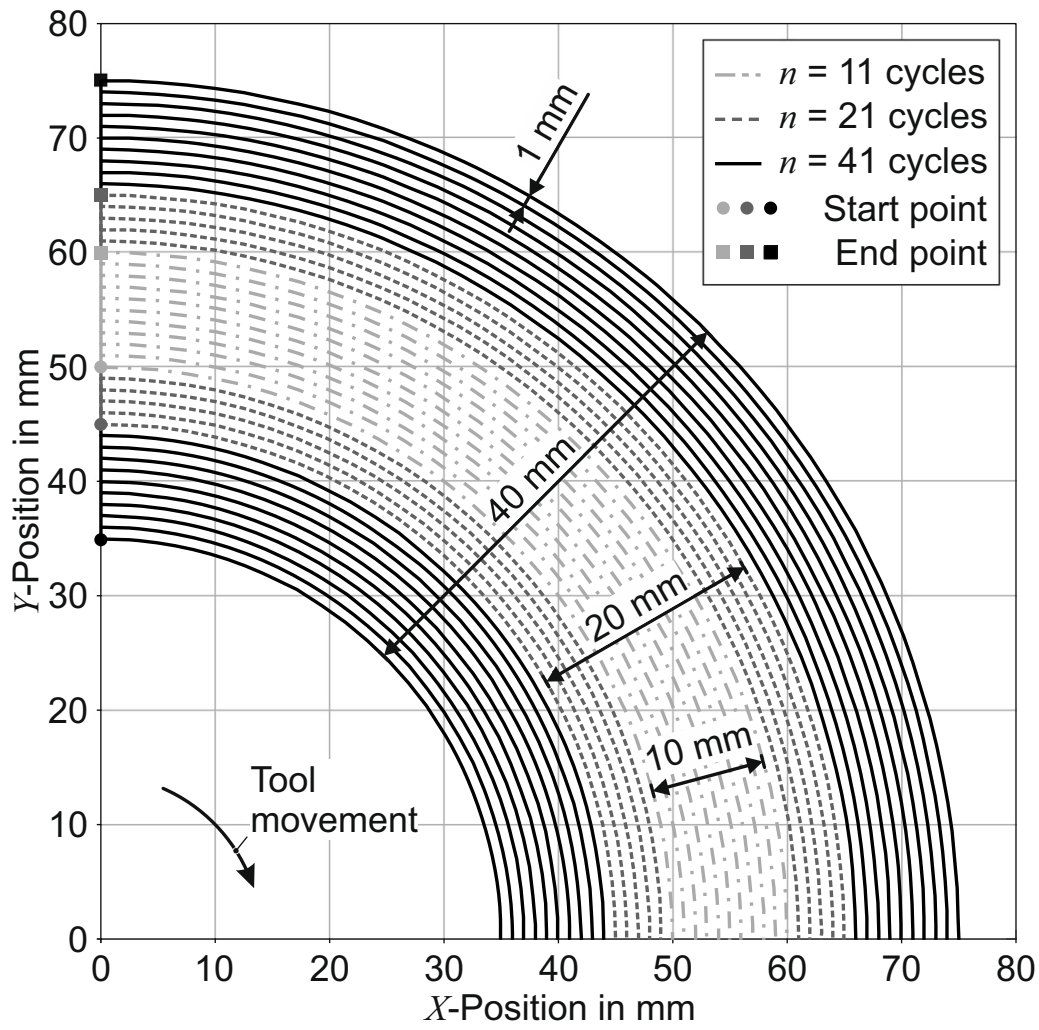


ANNs with many hidden layers, i.e. deep ANNs, were developed to learn complicated functions in high-dimensional spaces and overcome situations where traditional methods achieve unsatisfactory generalization rates. Deep networks, however, require a sufficiently large dataset to generate satisfactory results. The results of the exhaustive search support this notion, where ANNs with multiple hidden layers outperformed the ones with a singular hidden layer. The former will be further presented in "Offline pressure prediction" section.

\section{Control strategy}

The goal of the control is to ensure that a certain target height $h_{\mathrm{t}}(i)$ is achieved for each cycle $i$ during the manufacturing process. The primary goal of this control strategy is the improvement of the geometrical accuracy though the secondary goal to avoid cracks is accomplished at the same time. Therefore, it is classified as online closed-loop control of geometrical product properties. The control scheme is subdivided into a discrete control, which runs one time per forming cycle, and a continuous forming process (Fig. 4), which is denoted by the process time $t$. At the beginning of each cycle $i$, the target height $h_{\mathrm{t}}(i)$ that should be achieved after the cycle $i$ is compared with the actual height $h_{\mathrm{a}}(i-1)$ of the previous cycle (Eq. 2).

$$
\Delta h_{\mathrm{c}}(i)=h_{\mathrm{t}}(i)-\mathrm{h}_{\mathrm{a}}(i-1)
$$

The control deviation between them $\Delta h_{\mathrm{c}}(i)$ is the required growth of the part during the cycle $i$ so that the part shape follows a desired trajectory. The control then estimates the corresponding pressure $p(i)$ for the cycle $i$ using an ANN. With this strategy, not only an automated manufacturing process becomes possible but also disturbances $e_{\mathrm{P}}(t)$ can be compensated in subsequent cycles.
The control program is written in LabVIEW, and the ANN is integrated in the form of a Python function into its structure. The program reads the information concerning the target height $h_{\mathrm{t}}(i)$ and the circle diameter $d_{a}(i)$ of the tool path from a spreadsheet file. Additional settings regarding the part shape (small, middle, large) can be made before the experiments. A set of rules converts the actual $\mathrm{NC}$ block of the milling machine into the actual cycle $i$, and corresponding subroutines were carried out when the NC block changes. After each cycle, the measurement data is prepared so that the averaged force $F_{\mathrm{a}}(i-1)$ and the actual height $h_{\mathrm{a}}(i-1)$ of the completed cycle are available. From the perspective of control system engineering, this discrete working principle includes one dead cycle until the data is ready to use. To make sure that this data does not lead to invalid inputs or inappropriate outputs, both variables are limited to certain boundaries. The target height difference $\Delta h_{\mathrm{t}}(i)$ is restricted to a range from $0 \mathrm{~mm}$ to an adjustable value $\Delta h_{\max }$. This restriction avoids negative input values, which physically cannot exist for the convex forming process. Additionally, the input cannot grow to an unreasonable high value, which would require a high pressure and would lead to cracks. For the same reason, the output $p_{\mathrm{t}}(i)$ has an adjustable upper limit of $p_{\text {max }}$. The restrictions on the upper limit are motivated by process safety rather than control accuracy. The lower limit is fixed to 0.0 bar because a vacuum is not intended. The target pressure $p_{\mathrm{t}}(i)$ is then transmitted as continous signal $p_{\mathrm{t}}(t)$ to the pressure valve.

The time sequence of the control is depicted in Fig. 5. Both the milling machine and the LabVIEW program are synchronized by the help of NC blocks. Due to the dead cycle, there are differences between the process start, the periodic pressure adjustment and the process end. At the beginning, the tool moves in safe distance above the blank to the start positon in $X$ - and $Y$ coordinates. The start position is located $1 \mathrm{~mm}$ closer towards the center of blank than the radius of the first cycle. When the tool reaches the start position, a pressure is applied on the blank. Since

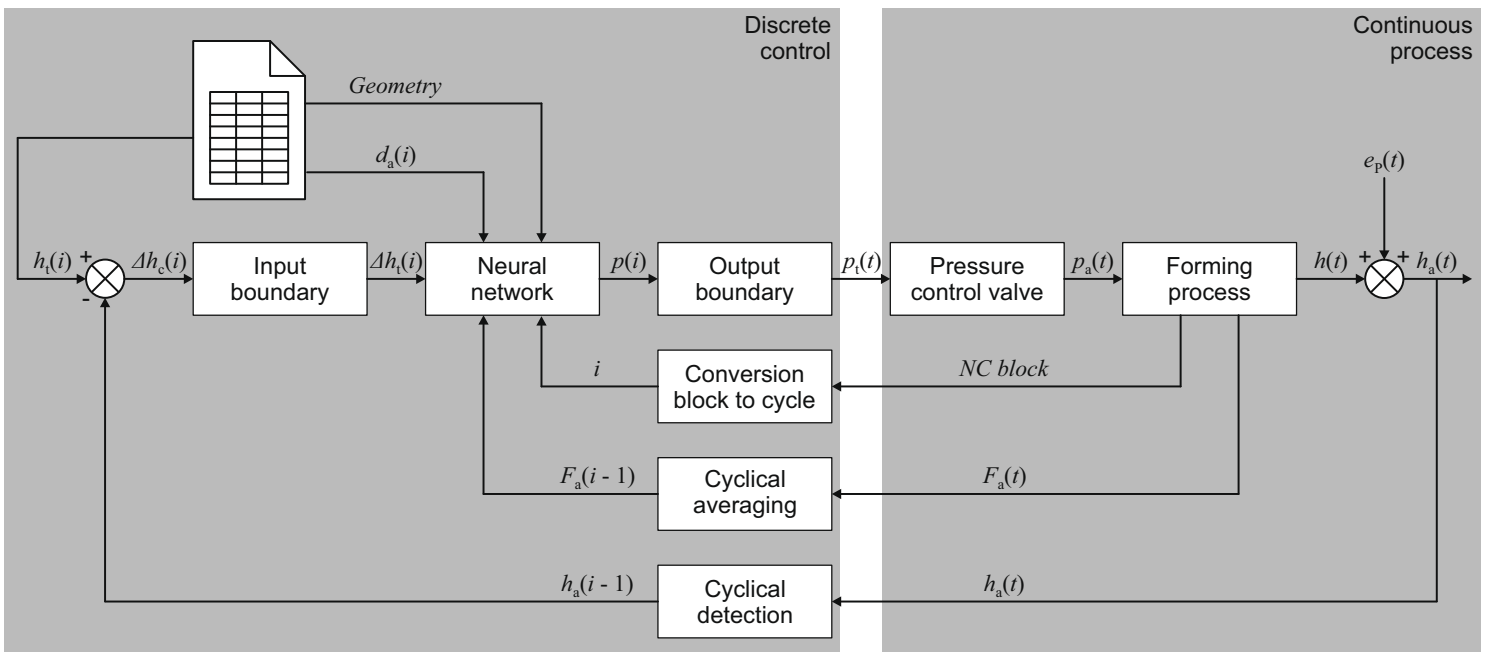

Fig. 4 Closed-loop control scheme to adjust the product height during IFAM 
Fig. 5 Three subroutines for the closed-loop control of IFAM: process start, periodic pressure adjustment between cycles and process end

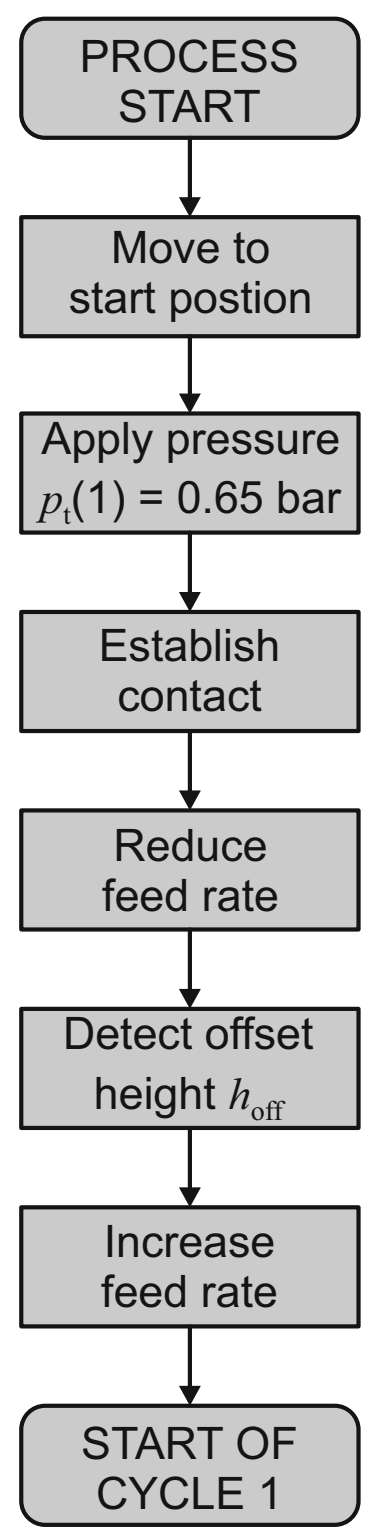

there are no process data available prior to the first cycle and the control algorithm cannot be executed, the pressure of the first cycle $p_{\mathrm{t}}(1)$ is set to 0.65 bar for all tests. Subsequently, the tool moves downwards with a feed rate $f_{\text {fast }}=1500 \mathrm{~mm} / \mathrm{min}$ until it reaches the vertical positon $z=0 \mathrm{~mm}$ and contact between the tool and the blank is established. The rotational speed does not change during the manufacturing process and is set to $60 \mathrm{rpm}$. The feed rate is then set to $f_{\text {slow }}=4 \mathrm{~mm} / \mathrm{min}$ so a period of $15 \mathrm{~s}$ remains to detect the reference height $h_{\text {off }}$ by averaging five measurement values. When the position of the first cycle is reached, the feed rate speeds up again and the forming operation begins. The target pressure $p_{t}(t)$ is constant throughout each cycle and the control system passively records the forming force $F_{\mathrm{a}}(t)$. At the end of each cycle, the feed rate slows down to give the control algorithm a time of $15 \mathrm{~s}$ until the next cycle starts. Within this time, the force $F_{\mathrm{a}}(i-1)$ is determined by averaging the recorded force values $F_{\mathrm{a}}(t)$ and the actual height $h_{\mathrm{a}}(i-1)$ is

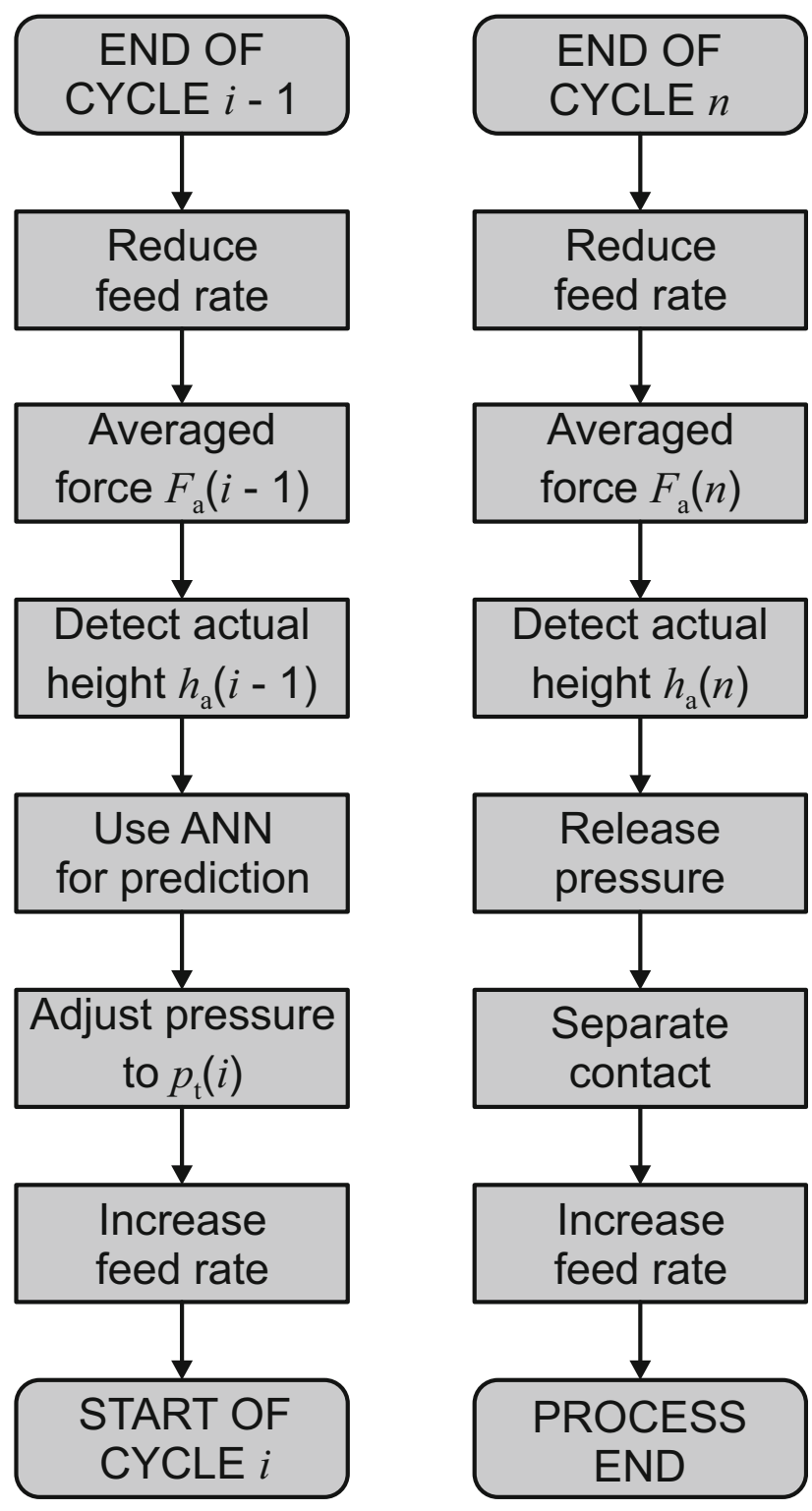

calculated as mean value of five current measurements of $h_{\mathrm{a}}(t)$. Afterwards, the control algorithm is executed including the boundary conditions to predict the pressure $p_{\mathrm{t}}(t)$ for the next cycle. Finally, the control valve adjusts the pressure inside the chamber according to this value. A new pressure value does not need to be calculated at the end of the last cycle. However, the data of this cycle are acquired while the tools moves slowly $1 \mathrm{~mm}$ towards the margin of the blank. In the final step, the pressure is released and the tool separates from the blank.

The controlling concept is evaluated by manufacturing truncated cones in three different sizes (Table 2). The narrow and wide parts according to Table 1 are only used for training purposes and are not considered for the controlling concept. The corresponding tool paths are similar to the ones described in Fig. 3 despite the fact that they have one cycle less. The final target height of large parts, for example, is defined as $h_{\mathrm{t}}(n)=70 \mathrm{~mm}$ with an ideal growth of $1.75 \mathrm{~mm}$ per cycle. 
Table 2 Tool path and target geometry definitions

\begin{tabular}{lllll}
\hline Part & Minor radius & Number of cycles $n$ & Target height $h_{\mathrm{t}}(n)$ & Wall angle $\alpha$ \\
\hline Small & $50 \mathrm{~mm}$ & 10 & $17.5 \mathrm{~mm}$ & $60.26^{\circ}$ \\
Middle & $45 \mathrm{~mm}$ & 20 & $35.0 \mathrm{~mm}$ & $60.26^{\circ}$ \\
Large & $35 \mathrm{~mm}$ & 40 & $70.0 \mathrm{~mm}$ & $60.26^{\circ}$ \\
\hline
\end{tabular}

(a)

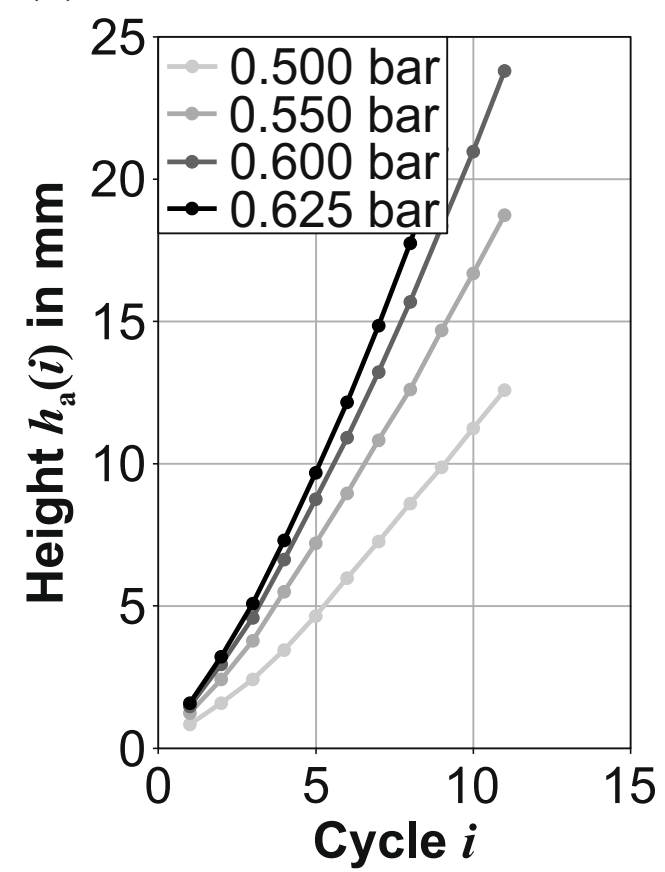

(b)

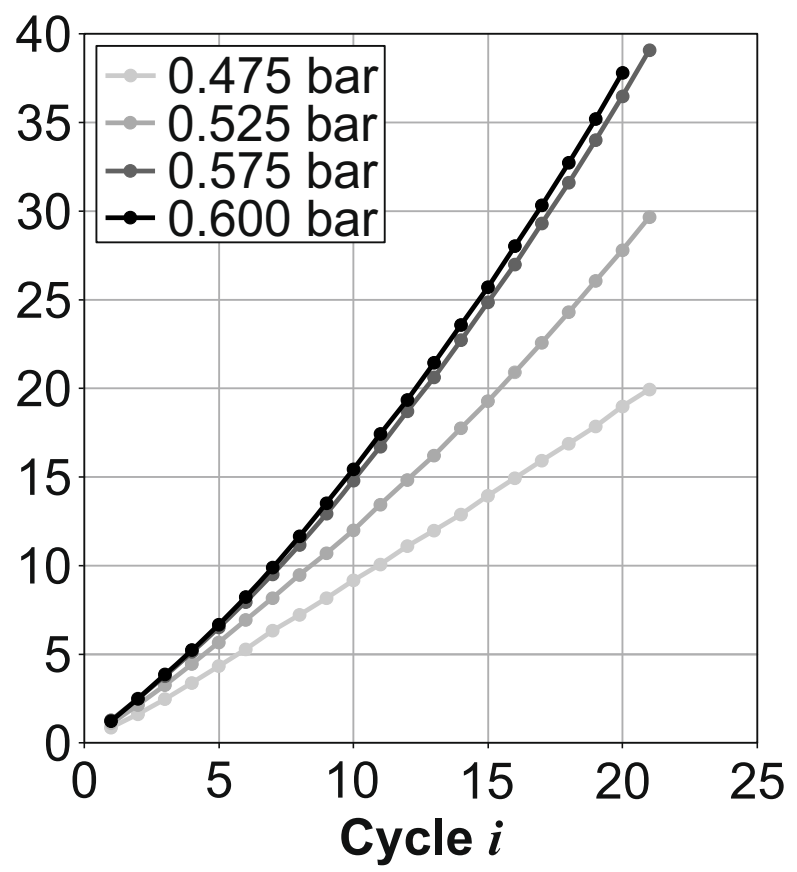

(c)

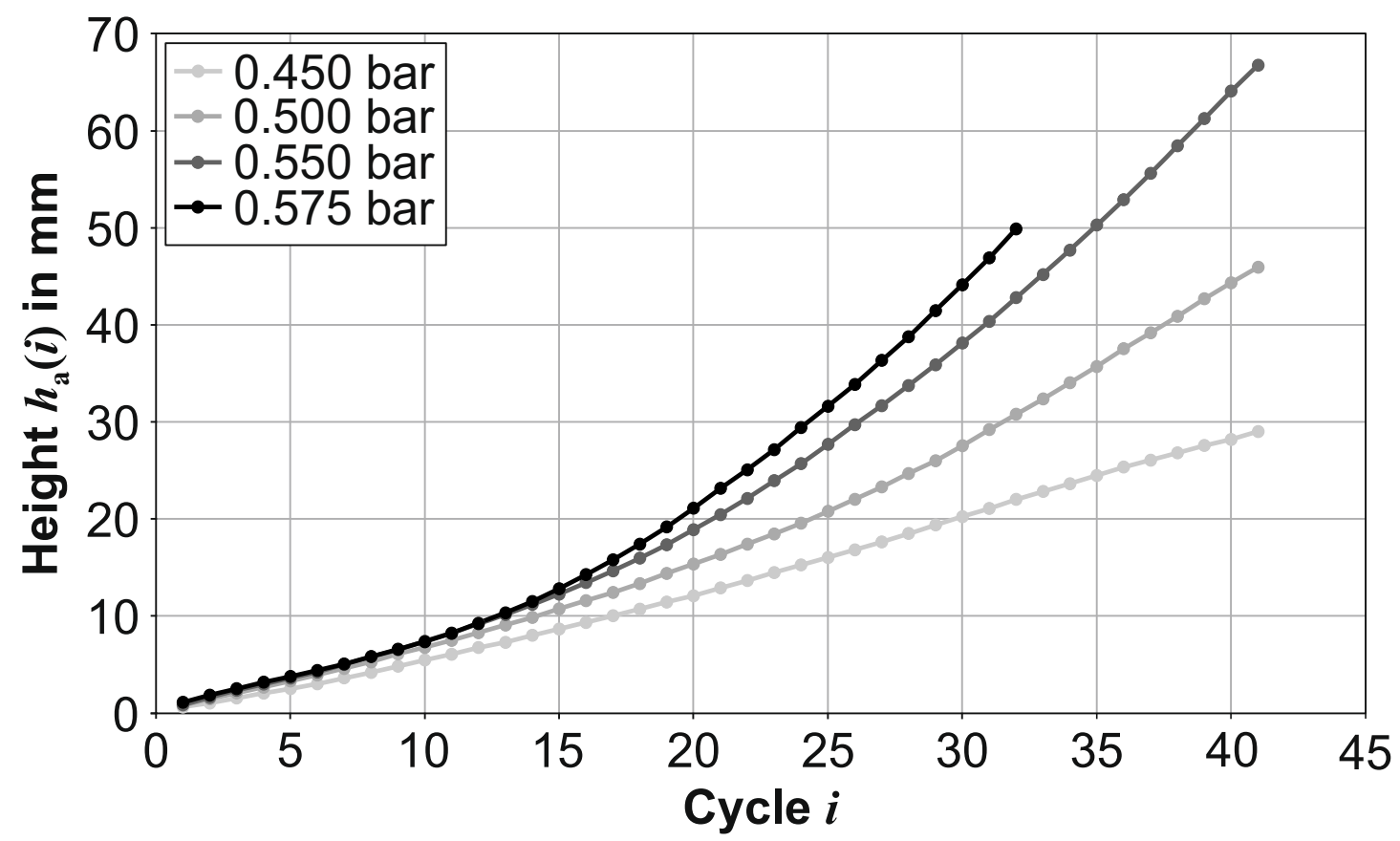

Fig. 6 Height growth $\boldsymbol{h}_{\mathbf{a}}(\boldsymbol{i})$ during the manufacturing process: a small parts $(\boldsymbol{n}=11)$, b middle-sized parts $(\boldsymbol{n}=21)$ and $\mathbf{c}$ large parts $(\boldsymbol{n}=41)$ 
Fig. 7 Change in height difference $\boldsymbol{\Delta} \boldsymbol{h}_{\mathrm{a}}(\boldsymbol{i})$ in IFAM: a small parts $(\boldsymbol{n}=11)$, b middlesized parts $(\boldsymbol{n}=21)$ and $\mathbf{c}$ large parts $(n=41)$

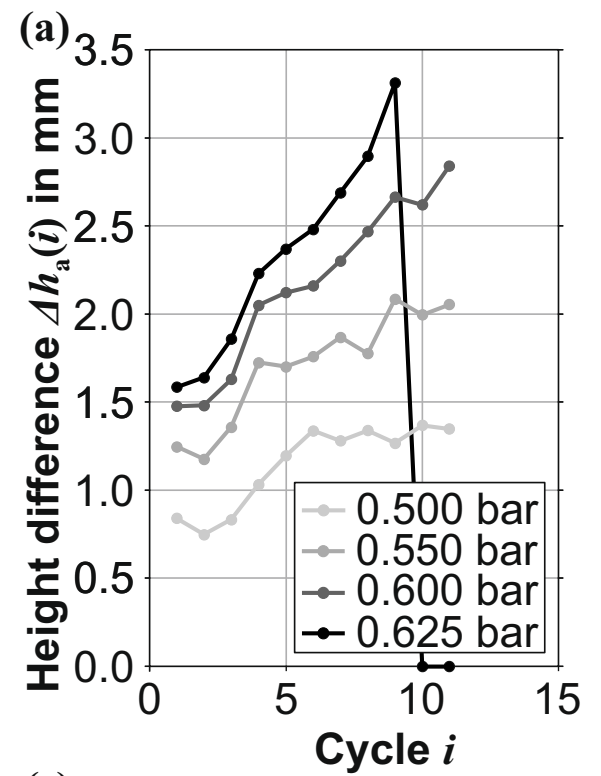

(b)

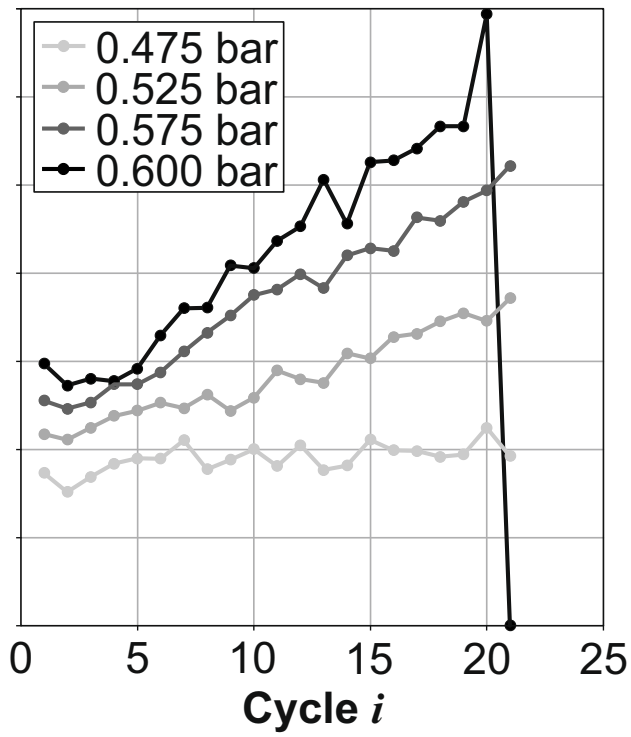

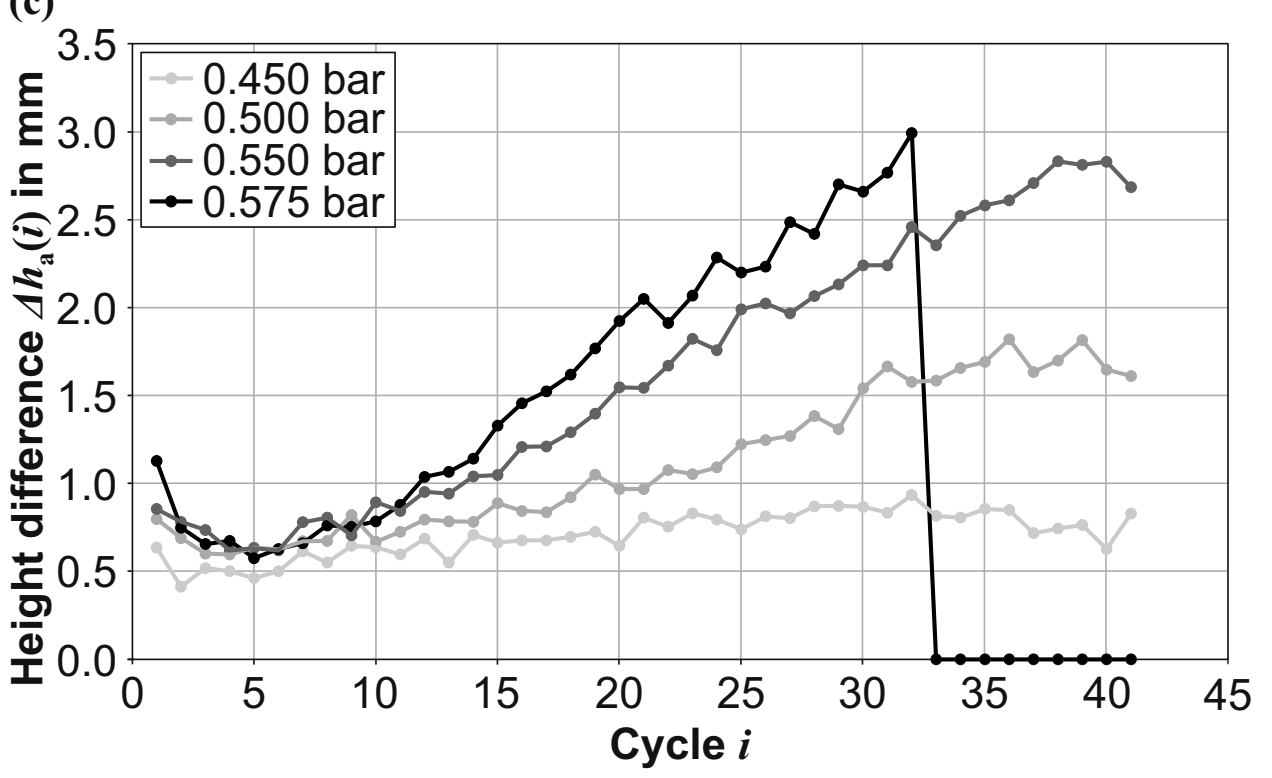

Only if the height difference $\Delta h_{\mathrm{a}}(i)$ during the manufacturing process is identical with this ideal growth, a conforming target wall angle of $60.26^{\circ}$ is achievable, which is equivalent for all parts. For these target geometries, the control strategy by using ANN is compared with the manufacturing process without control algorithm as reference.

\section{Results and discussion}

\section{Bulging behavior}

In this section, the bulging behavior of the different tool paths described in section 2.2 is summarized for a reduced selection of geometries and pressure levels. The development of the product height $h_{\mathrm{a}}(i)$ can be seen in Fig. 6. Fundamental findings indicate the great influence of the pressure $p$ and the number of cycles $n$ on the product height. Either a higher pressure or a higher number of cycles leads to a larger product height. However, when the pressure was set too high and cracks occurred, the forming process could not be continued and the parts did not achieve the maximum height that was obtainable with a lower pressure. Other than the curve of manufacturing a middle-sized part with a pressure of 0.475 bar, which has an almost homogeneous slope at any point, most curves are characterized by a non-linear trend with an increasing growth rate. The height difference $\Delta h_{\mathrm{a}}(i)$, Eq. 3, describes this issue in more detail and is shown in Fig. 7.

$\Delta h_{a}(i)=h_{\mathrm{a}}(i)-\mathrm{h}_{a}(i-1)$ 
Fig. 8 Development of the vertical forming force $\boldsymbol{F}_{\mathrm{a}}(\boldsymbol{i})$ in IFAM: a small parts $(\boldsymbol{n}=11), \mathbf{b}$ middle-sized parts $(\boldsymbol{n}=21)$ and $\mathbf{c}$ large parts $(n=41)$

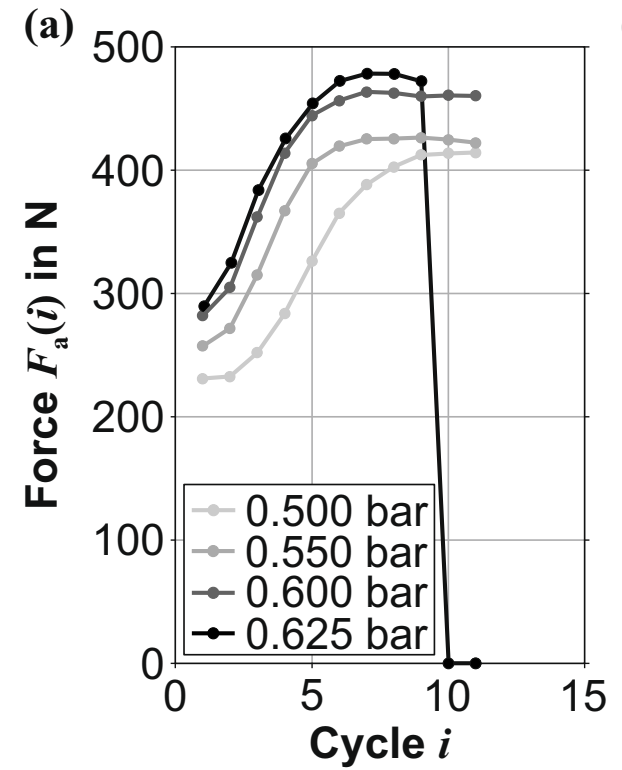

(b)

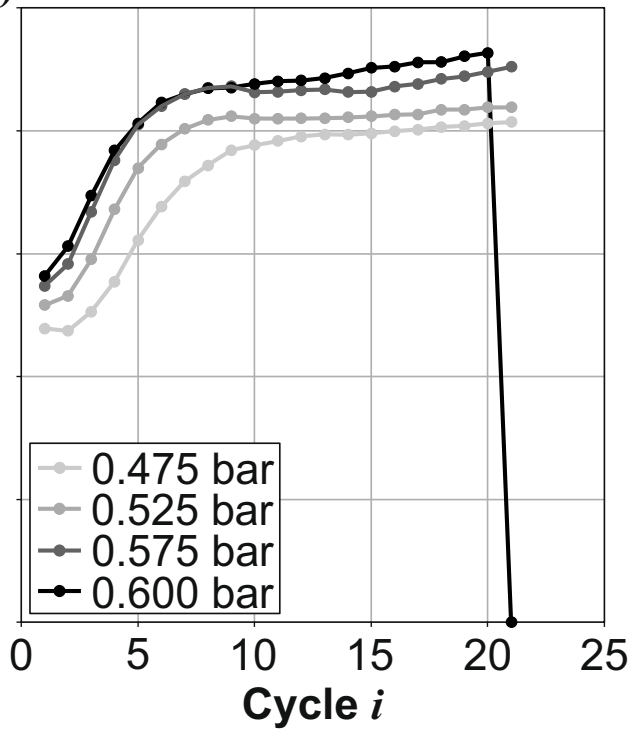

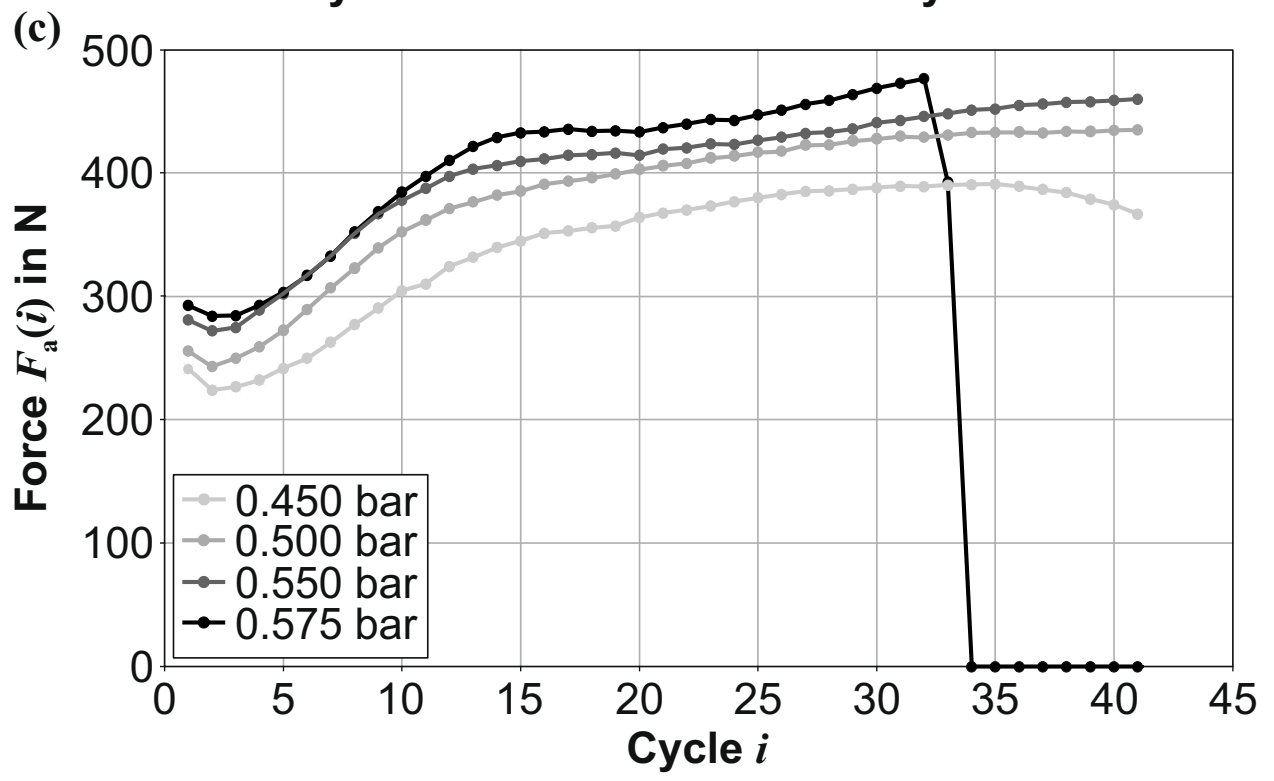

Especially, the small parts, Fig. 7a, and the middle-sized parts, Fig. $7 b$, show an almost linear development of the curve. Corresponding to Fig. 6b, the height difference does

Table 3 Number of samples within the dataset, before and after eliminating anomalies and extrapolation

\begin{tabular}{llll}
\hline Part & Original & $\begin{array}{l}\text { Non- } \\
\text { extrapolated }\end{array}$ & Extrapolated \\
\hline Small & 75 & 75 & 300 \\
Middle & 147 & 146 & 584 \\
Narrow & 147 & 146 & 584 \\
Wide & 147 & 139 & 556 \\
Large & 287 & 276 & 1104 \\
Total & 803 & 782 & 3128 \\
\hline
\end{tabular}

not increase for the pressure $p=0.475$ bar. In contrast, the large parts behave different at the beginning, where the tool start point is closer towards the center of the blank. The height

Table 4 Two consecutive cycles for a small-sized truncated cone after extrapolating the first cycle

\begin{tabular}{llllll}
\hline Geometry & $d_{\mathrm{a}}(i)$ & $i$ & $\Delta h_{\mathrm{a}}(i)$ & $F_{\mathrm{a}}(i)$ & $p_{\mathrm{t}}(i)$ \\
\hline Small & $\mathbf{1 0 0}$ & $\mathbf{1}$ & $\mathbf{0 . 7 8 1 9}$ & $\mathbf{2 2 6 . 1 4}$ & $\mathbf{0 . 4 7 5}$ \\
Small & 100 & 1 & 0 & 226.14 & 0.35 \\
Small & 100 & 1 & 0.391 & 226.14 & 0.4125 \\
Small & 100 & 1 & 1.1729 & 226.14 & 0.5375 \\
Small & $\mathbf{1 0 2}$ & $\mathbf{2}$ & $\mathbf{0 . 6 6 5 8}$ & $\mathbf{2 2 7 . 2 7}$ & $\mathbf{0 . 4 7 5}$ \\
\hline
\end{tabular}

Original cycles are shown in bold 
Fig. 9 Dataset for training the ANNs on the example of small truncated cones (Fig. 6a): a original data and $\mathbf{b}$ extrapolated data

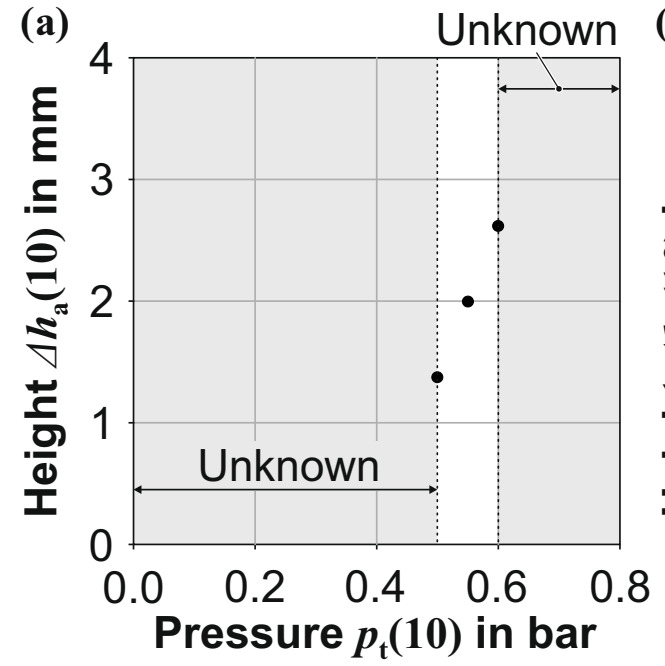

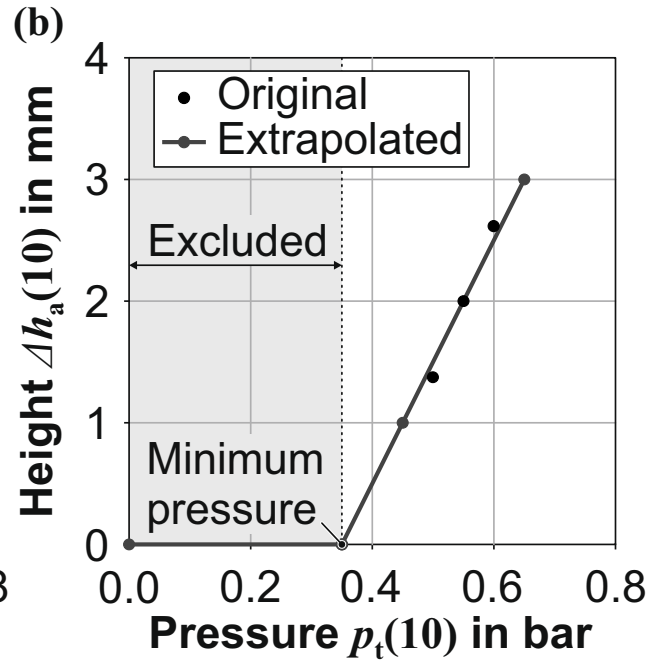

difference of the large parts is almost constant and lower than the height difference of the smaller parts within the first ten cycles. It can be deduced that the pressure has a lower influence on the height growth within these cycles. However, beyond the 10th cycle, the influence of the pressure is considerable and the height difference increases. Regarding all shapes, a high risk of cracks can be related to a combination of high pressure values and height differences $\Delta h_{\mathrm{a}}(i) \geq 3 \mathrm{~mm}$.

The development of the vertical force $F_{\mathrm{a}}(i)$ is depicted in Fig. 8 where, generally, a higher pressure causes a higher force. The force is initially in a range from $230 \mathrm{~N}$ to $300 \mathrm{~N}$ and increases strongly during the first ten cycles. After that, the forces curves flatten, stagnate or even decrease in some cases. The vertical force reaches values between $360 \mathrm{~N}$ and $460 \mathrm{~N}$ in the end of the manufacturing process. It can be concluded that in high pressure situations, forces $F_{\mathrm{a}}(i) \geq$ $460 \mathrm{~N}$ are an indicator for imminent cracks. Moreover, the force increases at a slower rate for larger parts than for the smaller ones. For example, in the fifth cycle where pressure $p=0.575$ bar, the force $F_{\mathrm{a}}(5)$ amounts to $304 \mathrm{~N}$ for a large part whereas it is already at $405 \mathrm{~N}$ for a middle-sized part. A possible explanation for this is the fact that the start point of

Table 5 ANN model parameters for the extrapolated dataset

\begin{tabular}{ll}
\hline Parameter & Value \\
\hline Hidden layers & 3 \\
Neurons per hidden layer & $500,250,150$ \\
Dropout ratio & 0.1 \\
Batch size & 32 \\
Optimizer & Adam \\
Learning rate & 0.001 \\
Iterations & 329 \\
Kernel initializer & Normal \\
\hline
\end{tabular}

the tool is located closer towards the blank's center and consequently the forming condition might be different there.

In summary, there is no linear or constant relationship between the product height $h_{\mathrm{a}}(i)$ and the pressure $p$. Additionally, the parameters are continuously changing throughout the manufacturing process, rendering it more prone to failure. Prior to using ANNs for learning the interrelations among the parameters, the data generated from the experiments is first described and undergoes pre-processing in the following section.

\section{Data description and extrapolation}

During training, the ANN used in order to predict pressure has five inputs and one output, namely: Geometry (Table 1), circle diameter of the tool path $d_{\mathrm{a}}(i)$, current cycle $i$, height difference $\Delta h_{\mathrm{a}}(i)$, average forming force $F_{\mathrm{a}}(i)$ and pressure $p_{\mathrm{t}}(i)$, respectively. While geometry is an ordinal categorical variable, all other variables and outputs are continuous. The original dataset consists in total of 803 observations for all geometries, as shown in Table 3. From there on, it undergoes a series of adjustments. First, invalid cycles within the dataset are removed. Invalid cycles are defined as instances after a crack appears in the part. On the one hand, these instances

Table 6 Performance across several metrics for: a ANN nonextrapolated data and $\mathbf{b}$ ANN extrapolated data $\mathbf{c}$ linear regression $\mathbf{d}$ polynomial regression

Model $\mathrm{R}^{2}$ train $\mathrm{R}^{2}$ test MSE train MSE test MAE train MAE test

\begin{tabular}{lllllll}
\hline$a$ & 0.962 & 0.94 & 0.0018 & 0.0028 & 0.024 & 0.032 \\
$b$ & 0.984 & 0.978 & 0.0008 & 0.0011 & 0.015 & 0.018 \\
$c$ & 0.76 & 0.761 & 0.0124 & 0.138 & 0.074 & 0.081 \\
$d$ & 0.964 & 0.967 & 0.0017 & 0.002 & 0.023 & 0.026 \\
\hline
\end{tabular}




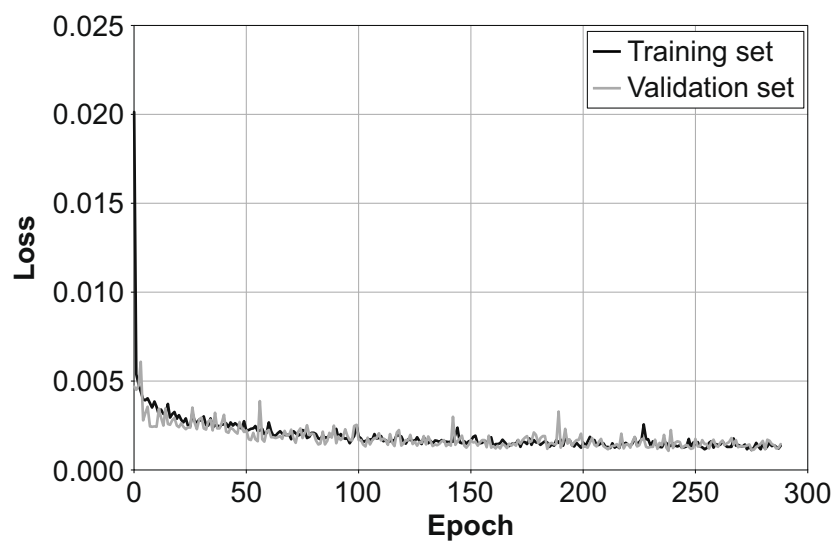

Fig. 10 Model $b$ learning curve; the development of MSE with increasing epochs

give rise to false measurements of the height, and on the other hand, the forming process cannot be continued once a crack appears, since the pressure in the pressure chamber can no longer be maintained. A benchmark ANN is trained using the remaining 782 observations and compared with a better performing one that is trained with extrapolated data. The reasons behind extrapolating are two-fold: first, to project and extend the known pressure-height relationship into areas which are not covered by the experiments, and second, to generate more observations. For all points, the extrapolation occurs linearly and begins at $p=0.35$ bar, which is considered to be the minimum pressure at which deformation can take place for pure Aluminum, as used with comparable hardening states in [1] and in the experiments conducted in this work. This procedure can be seen in Fig. 9, as well as in Table 4 . Though the extrapolation is linear, it was only performed on each cycle independently. Consequently, it does not affect the non-linear relationship between the product height $h_{\mathrm{a}}(i)$ and the pressure $p$.

After extrapolating, the data contains 3128 observations to be used for training and testing purposes. The input and output variables were normalized using the max-min normalization method before training. Each independent variable $x$ was mapped to the range $[-1,1]$ through the transformation by the expression:

$\frac{\left(x-x_{\min }\right)}{\left(x_{\max }-x_{\min }\right)} *(\max -\min )+\min$

Where min, max are the defined feature ranges, $x$ the current value and $x_{\min }, x_{\max }$ the minimum and maximum value in the dataset respectively. Similarly, each independent variable $y$ was mapped to the range $[0,1]$.

\section{Offline pressure prediction}

In the case presented in the paper, where the target is a continuous variable, the mean squared error (MSE) is chosen as the loss function to be minimized. MSE measures the average square difference of the experimental (actual) value and the value predicted by the ANN. Additionally, the non-linear activation function $\sigma$ for the input and hidden layers is selected to be the Rectified Linear Unit (ReLU), with the final output layer undergoing no transformation. ReLU converges relatively fast and is efficient in multiplying gradients over multiple layers [27], which makes it one of the most widely used non-linear activation functions for deep networks. The exhaustive search showed that the best performing model consists of 3 hidden layers with 500, 250, and 150 neurons respectively in each layer. Further hyperparameters are presented in Table 5. To properly estimate the generalization error of the learner, the dataset is split into three subsets, namely training (90\%), validation (5\%) and testing (5\%). The test set is not involved in training the learner, and is only called after training to estimate the true generalization error of the algorithms as best as possible. The model is ultimately trained using the listed hyperparameters 25 times, where the data is re-shuffled for every replication, resulting in a different train / test split for every run. The latter is performed to account for the stochastic nature of neural networks. The average of these 25 runs for the model trained on the extrapolated dataset is shown in Table 6b. A different ANN model was trained on nonextrapolated data and serves as a benchmark to compare the results with (Table 6a). This model undergoes an exhaustive search as well, which results in a different set of hyperparameters to be used. Moreover, other machine learning models such as linear regression and polynomial regression were found to be less accurate, as depicted in Table $6 \mathrm{c}$, d. The models achieved satisfactory results across multiple performance metrics, with model $b$ outperforming its counterparts. The relatively high value of the coefficient of determination $\mathrm{R}^{2}=0.984$ achieved indicates that the model explains the variance of $p$ with respect to the input parameters sufficiently well. The mean absolute error (MAE) presented for the test set shows that on average, a prediction of a new pressure point in the range of $[0,1]$ deviates 0.018 bar from the real value of said pressure point. Finally, it can be deduced from Fig. 10 that model $b$ is not overfit on the training data; the loss (MSE) decrease for the training and validation sets is relatively close, and the model can thus generalize its learning on samples it has not come across before. Therefore, model $b$ is selected to be used in the following sections for controlling the pressure levels.

\section{Influence on geometrical accuracy}

The geometrical accuracy consists of the height difference of each cycle along with the final accumulated height. At first, the development of the height difference during the manufacturing process is analyzed for constant and dynamically adjusted pressure, in Figs. 11 and 12 respectively. 

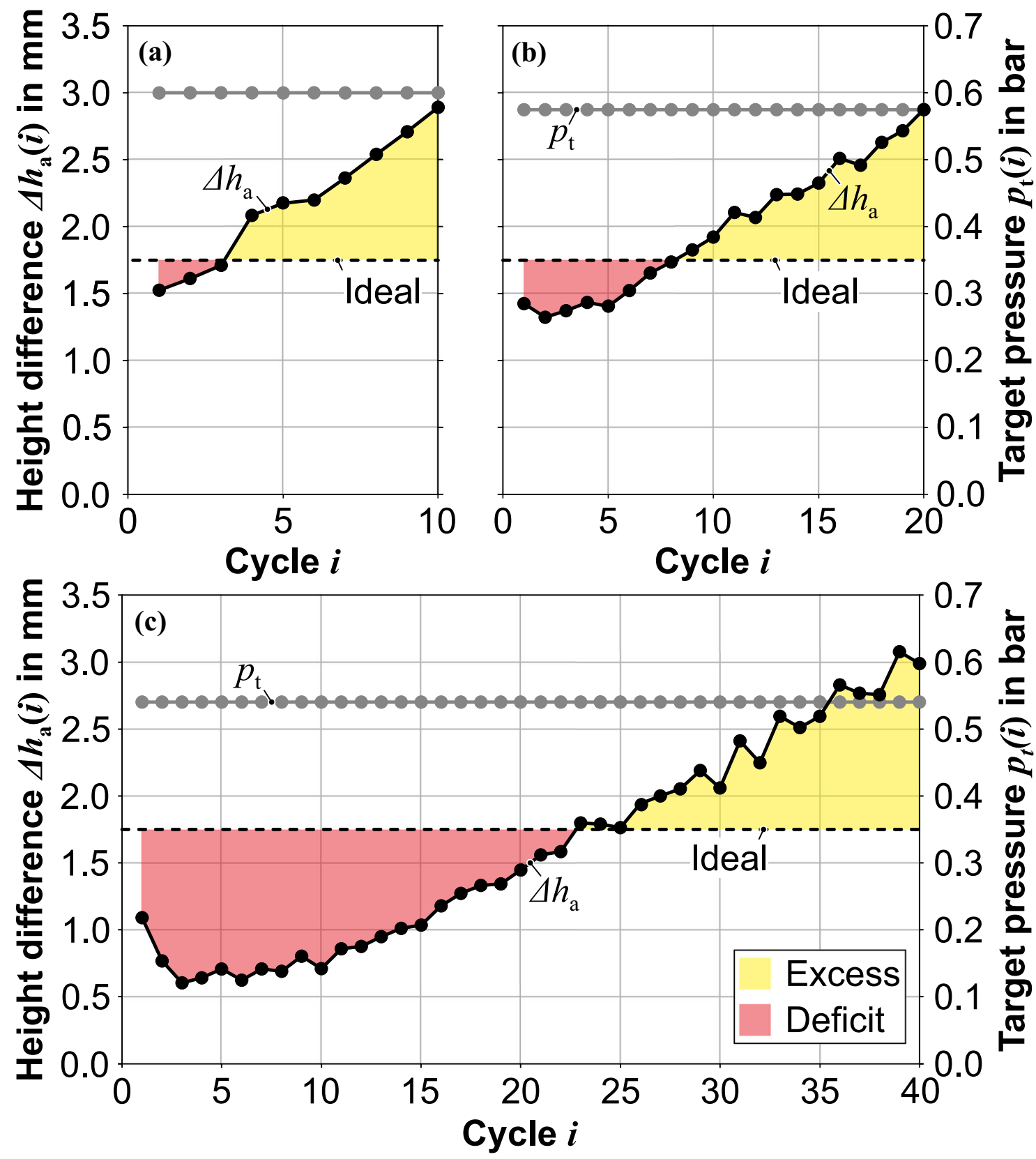

Fig. 11 Development of the height difference $\Delta \boldsymbol{h}_{\mathrm{a}}(\boldsymbol{i})$ with constant pressure $\boldsymbol{p}_{\boldsymbol{t}}(\boldsymbol{i})$ for the uncontrolled forming process: a small part $(\boldsymbol{n}=10)$, $\mathbf{b}$ middlesized part $(\boldsymbol{n}=20)$ and $\mathbf{c}$ large part $(\boldsymbol{n}=40)$

Ideally, the height difference $\Delta h_{\mathrm{a}}(i)$ should be $1.75 \mathrm{~mm}$ for each cycle. If it is not the case, then the final height would either be exceeded or not achieved. For the uncontrolled process, the highest pressure levels from Table 1 which did not lead to cracks were selected as constant setpoints. From Fig. 11, it can be seen that the height difference starts of smaller than the value of $1.75 \mathrm{~mm}$ for all parts. It increased continuously to reach values exceeding $1.75 \mathrm{~mm}$ thereafter. The height difference had also reached the critical value of $3 \mathrm{~mm}$ during the manufacturing of the large part. As for the case where pressure was dynamically adjusted, it can be seen in Fig. 12 that any deficit or excess of the height difference was compensated by the controller. Furthermore, the control concept helped prevent the height difference from exceeding the critical value of $3 \mathrm{~mm}$ for all geometries.

The comparison of the final height for parts manufactured using constant and dynamically adjusted pressure is shown in Fig. 13, where the final contour was measured by a 3D-scanner. In the case of constant pressure, the small and middlesized parts exceeded the target height value whereas the large part remained well below it. Using dynamically adjusted pressure, all parts underwent significant improvements in terms of accuracy. It is also worth noting that the final height reached was consistently slightly above the target height. This is 

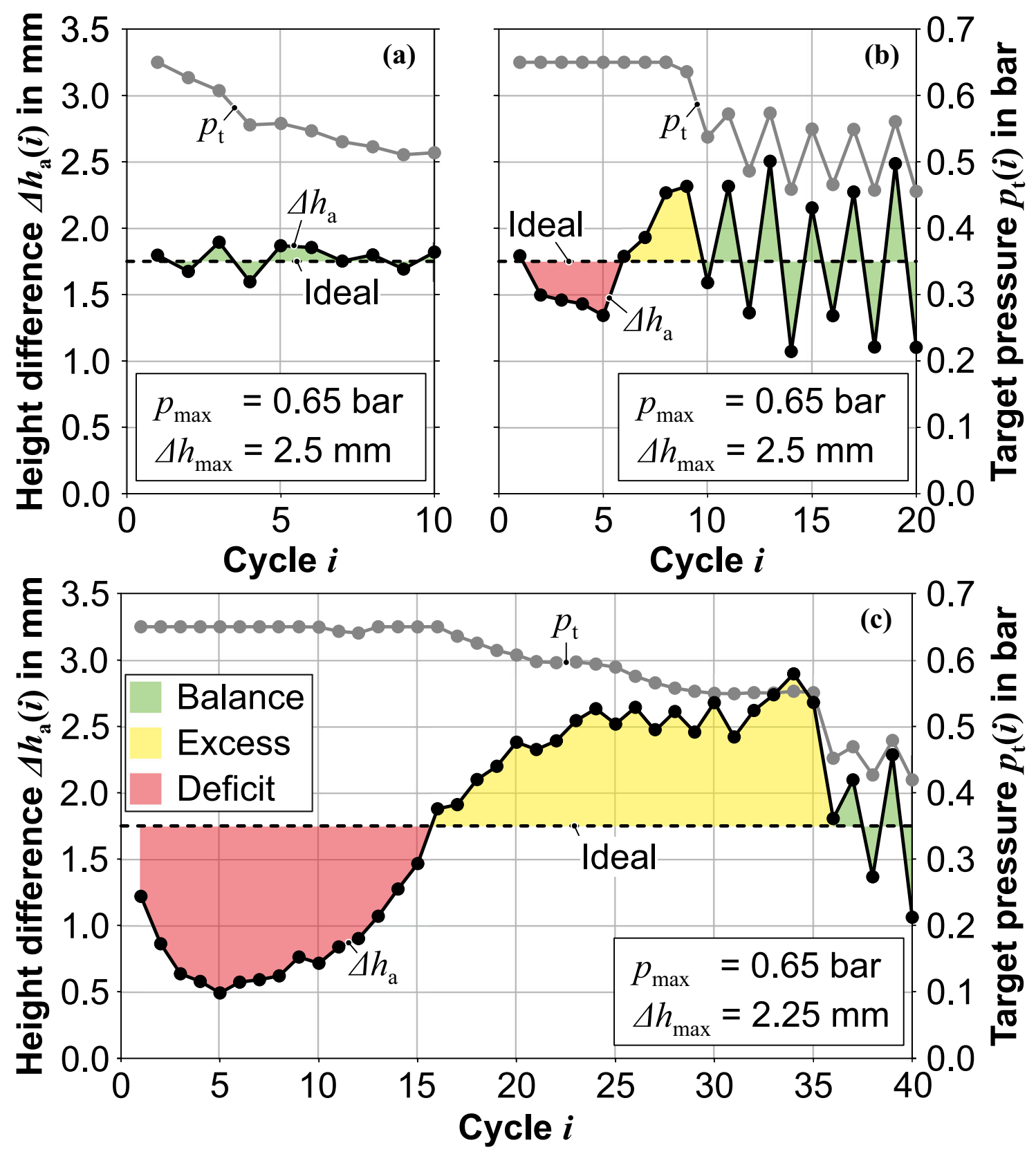

Fig. 12 Development of the height difference $\Delta \boldsymbol{h}_{\mathrm{a}}(\boldsymbol{i})$ and the target pressure $\boldsymbol{p}_{\mathrm{t}}(\boldsymbol{i})$ for the controlled forming process by using ANN: a small part $(\boldsymbol{n}=10)$, b middle-sized part $(\boldsymbol{n}=20)$ and $\mathbf{c}$ large part $(\boldsymbol{n}=40)$

caused by elastic bulging which occurs at the beginning of the forming process along with springback at the end after

Table 7 Elastic bulging prior to the first cycle and springback after pressure release as average value for all geometries

\begin{tabular}{llll}
\hline & Small $(n=10)$ & Middle $(n=20)$ & Large $(n=40)$ \\
\hline Elastic bulging & $3.2 \mathrm{~mm}$ & $2.8 \mathrm{~mm}$ & $2.6 \mathrm{~mm}$ \\
Springback & $-1.1 \mathrm{~mm}$ & $-1 \mathrm{~mm}$ & $-0.7 \mathrm{~mm}$ \\
$\sum=$ & $2.1 \mathrm{~mm}$ & $1.8 \mathrm{~mm}$ & $1.9 \mathrm{~mm}$ \\
\hline
\end{tabular}

pressure release in the chamber. These phenomena lead to a mismatch between the height measured by the laser sensor and the height determined by the 3D-scanner. Ideally, this mismatch can be compensated through adjustment of the initial height $h_{\text {off }}$ according to the values in Table 7 , which was not performed for the experiments.

By controlling the product height $h_{\mathrm{a}}(i)$, the wall angle $\alpha$ was indirectly influenced. The wall angles $\alpha$ corresponding to the cross sections of Fig. 13 are shown in Fig. 14. The wall angle never remained at a constant value without the control and also exceeded the target value of $60.26^{\circ}$ defined in Table 2 . In contrast, the controlled process led to a wall angle 
(a)

(b)

(c)
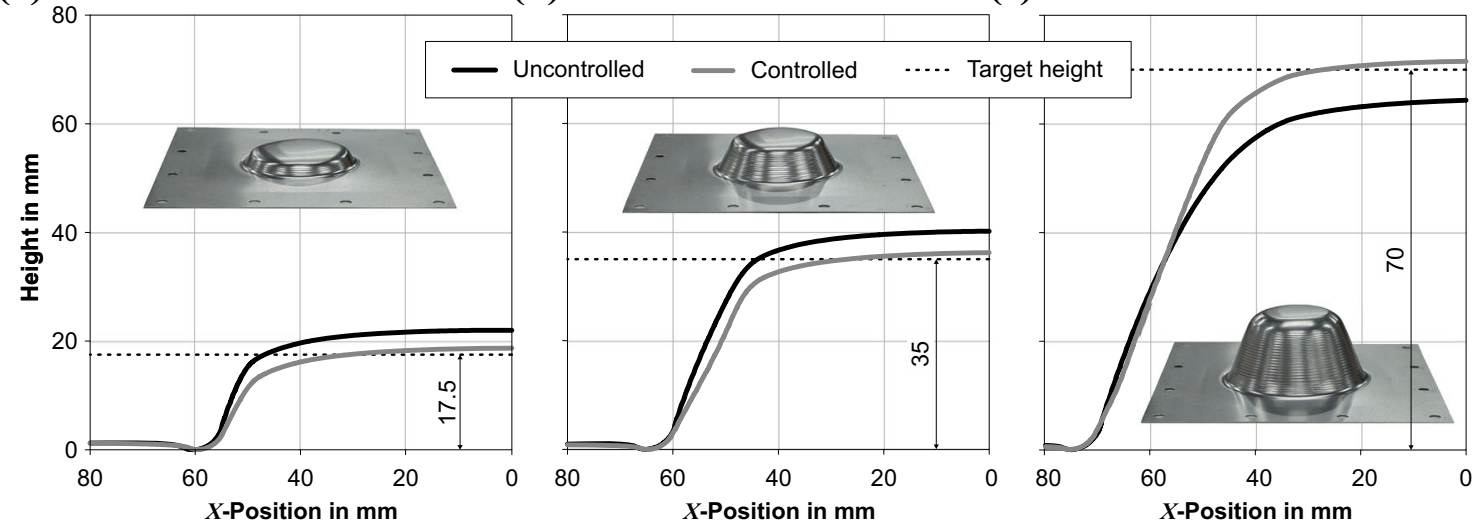

Fig. 13 Resulting product shape compared between the controlled case with automatically adjusted pressure and the uncontrolled case with constant pressure: a small parts $(n=10)$, b middle-sized parts $(n=20)$ and $\mathbf{c}$ large parts $(n=40)$; photos show the controlled parts

which is more in agreement with the target value. Thus, controlling the pressure can lead to more homogenous sheet thinning in the part wall.

\section{Conclusions}

In this work, a strategy was implemented to control the final part height in incremental sheet forming with active medium. Experiments were conducted to form truncated cones of different geometries and generate a dataset that was used to train an artificial neural network. Further data was created through extrapolating the pressure-height values of the original dataset. The neural network was part of a larger closed-loop control, in which the pressure for each forming cycle is to be predicted. The results were validated both through evaluating the performance of the neural network on a separate test set and through conducting experiments where the entire closedloop control was used to shape parts of different geometries. Finally, the parts formed using the closed-loop control were compared against uncontrolled parts manufactured using constant pressure. The approach followed by the authors, including conclusions drawn from the results are summarized as follows:

Fig. 14 Comparison of the wall angle $\boldsymbol{\alpha}$ between the uncontrolled forming process and the controlled forming process by using ANN: a small part $(\boldsymbol{n}=10)$, b middle-sized part $(\boldsymbol{n}=20)$ and $\mathbf{c}$ large part $(n=40)$

- Controlling the process through dynamically adjusting the pressure levels improves the geometrical accuracy. The final height, the development of the height difference of each cycle and the wall angle were all more in agreement with the target values. Uncontrolled processes with constant pressure were found to be less accurate.

- Both the elastic bulging which occurs prior to the process start and the springback which occurs after the pressure is released influence the final height of the part. They must be therefore taken into consideration in order to achieve the desired part height accurately.

- A combination of a uniform product height increase, a homogeneous wall angle distribution and a regulated decrease of the pressure contributes to a more reliable manufacturing process.

Furthermore, the approach followed in this work can be applied to any different material, so long as sufficient experiments are conducted and an adequate training dataset generated. In future work, the authors will look into further generalizing the closed-loop control approach to be applicable for different types of materials and geometries, such as for truncated pyramids. The authors will therefore investigate the minimum number of experiments required to be able to

(b)

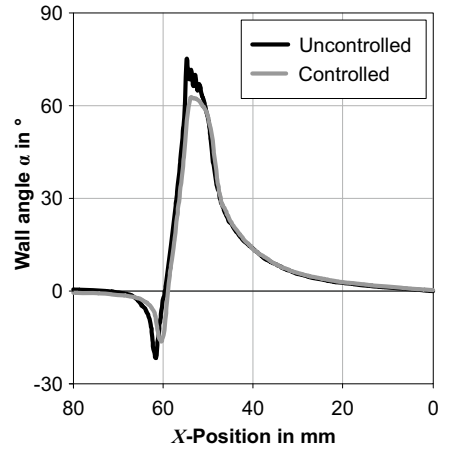

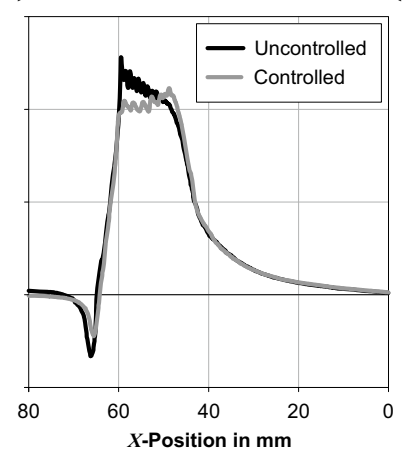

(c)

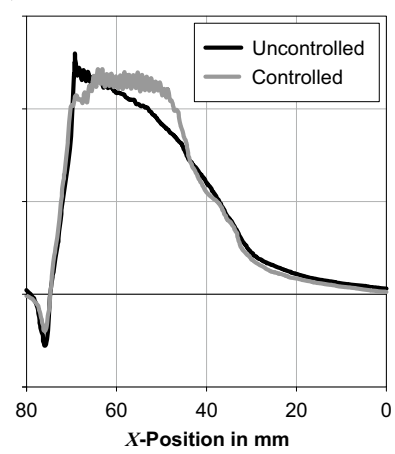


control the process reliably. Moreover, the exhaustive grid search which was used to find the best hyperparameters for the neural network was found to be time consuming and as such will be replaced by a method which converges faster, for example Bayesian optimization.

Acknowledgments The authors would like to thank the German Research Foundation (DFG) for supporting this research project under the grant number BE 5196/14-1.

Funding Open Access funding enabled and organized by Projekt DEAL.

Data availability The raw/processed data required to reproduce these findings cannot be shared at this time as the data also forms part of an ongoing study.

\section{Compliance with ethical standards}

Conflict of interest The authors declare that they have no conflict of interest.

Open Access This article is licensed under a Creative Commons Attribution 4.0 International License, which permits use, sharing, adaptation, distribution and reproduction in any medium or format, as long as you give appropriate credit to the original author(s) and the source, provide a link to the Creative Commons licence, and indicate if changes were made. The images or other third party material in this article are included in the article's Creative Commons licence, unless indicated otherwise in a credit line to the material. If material is not included in the article's Creative Commons licence and your intended use is not permitted by statutory regulation or exceeds the permitted use, you will need to obtain permission directly from the copyright holder. To view a copy of this licence, visit http://creativecommons.org/licenses/by/4.0/.

\section{References}

1. Ben Khalifa N, Thiery S (2019) Incremental sheet forming with active medium. CIRP Ann Manuf Technol 68(1):313-316. https://doi.org/10.1016/j.cirp.2019.04.043

2. Kumar Y, Kumar S (2018) Analysis of pressure assisted incremental sheet forming process through simulation. Int J Mech Prod Eng Res Dev 8:921-932. https://doi.org/10.24247/ijmperdjun201898

3. McLoughlin K, Cognot A, Quigley E (2003) Dieless manufacturing of sheet metal components with non rigid support. Proc SheMet 2003:123-130

4. Kumar Y, Kumar S (2019) Experimental and analytical evaluation of incremental sheet hydro-forming strategies to produce high forming angle sheets. Heliyon 5(6):e01801. https://doi.org/10. 1016/j.heliyon.2019.e01801

5. Junk S (2003) Inkrementelle Blechumformung mit CNCWerkzeugmaschinen: Verfahrensgrenzen und Umformstrategien. Saarland University, Saarbruecken

6. Ndip-Agbor E, Ehmann K, Cao J (2018) Automated flexible forming strategy for geometries with multiple features in doublesided incremental forming. J Manuf Sci Eng 140(3):88. https://doi. org/10.1115/1.4038511

7. Bambach M (2008) Process strategies and modelling approaches for asymmetric incremental sheet forming. RWTH Aachen, Aachen
8. Hirt G, Ames J, Bambach M, Kopp R, Kopp R (2004) Forming strategies and process modelling for $\mathrm{CNC}$ incremental sheet forming. CIRP Ann Manuf Technol 53(1):203-206. https://doi. org/10.1016/S0007-8506(07)60679-9

9. Ambrogio G, Costantino I, de Napoli L, Filice L, Fratini L, Muzzupappa M (2004) Influence of some relevant process parameters on the dimensional accuracy in incremental forming: a numerical and experimental investigation. J Mater Process Technol 153154:501-507. https://doi.org/10.1016/j.jmatprotec.2004.04.139

10. Endelt B (2017) Design strategy for optimal iterative learning control applied on a deep drawing process. Int J Adv Manuf Technol 88(1-4):3-18. https://doi.org/10.1007/s00170-016-8501-z

11. Calmano S, Hesse D, Hoppe F, Groche P (2015) Evaluation of control strategies in forming processes. MATEC Web Conf 21: 4002. https://doi.org/10.1051/matecconf/20152104002

12. Allwood JM, Duncan SR, Cao J, Groche P, Hirt G, Kinsey B, Kuboki T, Liewald M, Sterzing A, Tekkaya AE (2016) Closedloop control of product properties in metal forming. CIRP Ann Manuf Technol 65(2):573-596. https://doi.org/10.1016/j.cirp. 2016.06.002

13. Allwood JM, Music O, Raithathna A, Duncan SR (2009) Closedloop feedback control of product properties in flexible metal forming processes with mobile tools. CIRP Ann Manuf Technol 58(1):287-290. https://doi.org/10.1016/j.cirp.2009.03.065

14. Filice L, Ambrogio G, Micari F (2006) On-line control of single point incremental forming operations through punch force monitoring. CIRP Ann Manuf Technol 55(1):245-248. https://doi.org/10. 1016/S0007-8506(07)60408-9

15. Tao F, Qi Q, Liu A, Kusiak A (2018) Data-driven smart manufacturing. J Manuf Syst 48:157-169. https://doi.org/10. 1016/j.jmsy.2018.01.006

16. Chen JC, Chen JC (2005) An artificial-neural-networks-based inprocess tool wear prediction system in milling operations. Int $\mathrm{J} \mathrm{Adv}$ Manuf Technol 25(5-6):427-434. https://doi.org/10.1007/s00170003-1848-y

17. Pal S, Heyns PS, Freyer BH, Theron NJ, Pal SK (2011) Tool wear monitoring and selection of optimum cutting conditions with progressive tool wear effect and input uncertainties. J Intell Manuf 22(4):491-504. https://doi.org/10.1007/s10845-009-0310-x

18. Kar AK (2015) A hybrid group decision support system for supplier selection using analytic hierarchy process, fuzzy set theory and neural network. J Comput Sci 6:23-33. https://doi.org/10.1016/j. jocs.2014.11.002

19. Radetzky M, Rosebrock C, Bracke S (2019) Approach to adapt manufacturing process parameters systematically based on machine learning algorithms. IFAC-PapersOnLine 52:1773-1778. https:// doi.org/10.1016/j.ifacol.2019.11.458

20. Essid O, Laga H, Samir C (2018) Automatic detection and classification of manufacturing defects in metal boxes using deep neural networks. PLoS One 13(11):e0203192. https://doi.org/10.1371/ journal.pone.0203192

21. Heger J, Zein El Abdine M (2019) Using data mining techniques to investigate the correlation between surface cracks and flange lengths in deep drawn sheet metals. IFAC-PapersOnLine 52(13): 851-856. https://doi.org/10.1016/j.ifacol.2019.11.236

22. Khan MS, Coenen F, Dixon C, el-Salhi S, Penalva M, Rivero A (2015) An intelligent process model: predicting springback in single point incremental forming. Int J Adv Manuf Technol 76(9-12): 2071-2082. https://doi.org/10.1007/s00170-014-6431-1

23. Hartmann C, Opritescu D, Volk W (2019) An artificial neural network approach for tool path generation in incremental sheet metal free-forming. J Intell Manuf 30(2):757-770. https://doi.org/10. 1007/s10845-016-1279-x

24. Kurra S, Hifzur Rahman N, Regalla SP, Gupta AK (2015) Modeling and optimization of surface roughness in single point 
incremental forming process. J Mater Res Technol 4(3):304-313. https://doi.org/10.1016/j.jmrt.2015.01.003

25. Ambrogio G, Filice L, Guerriero F, Guido R, Umbrello D (2011) Prediction of incremental sheet forming process performance by using a neural network approach. Int J Adv Manuf Technol 54(9-12):921-930. https://doi.org/10.1007/ s00170-010-3011-x

26. Schwarzer M, Rogan B, Ruan Y, Song Z, Lee DY, Percus AG, Chau VT, Moore BA, Rougier E, Viswanathan HS, Srinivasan G (2019) Learning to fail: predicting fracture evolution in brittle material models using recurrent graph convolutional neural networks. Comput Mater Sci 162:322-332. https://doi.org/10.1016/j. commatsci.2019.02.046

27. Krizhevsky A, Sutskever I, Hinton GE (2017) ImageNet classification with deep convolutional neural networks. Commun ACM 60(6):84-90. https://doi.org/10.1145/3065386

Publisher's note Springer Nature remains neutral with regard to jurisdictional claims in published maps and institutional affiliations. 